\title{
On the Essential Spectrum of Many-Particle Pseudorelativistic Hamiltonians with Permutational Symmetry Account
}

\author{
Grigorii ZHISLIN
}

Radiophysical Research Institute, 25/14 Bol'shaya Pechorskaya Str., Nizhny Novgorod, 603950 Russia

E-mail: greg@nirfi.sci-nnov.ru

Received October 27, 2005, in final form February 07, 2006; Published online February 20, 2006

Original article is available at http://www.emis.de/journals/SIGMA/2006/Paper024/

\begin{abstract}
In this paper we formulate our results on the essential spectrum of many-particle pseudorelativistic Hamiltonians without magnetic and external potential fields in the spaces of functions, having arbitrary type $\alpha$ of the permutational symmetry. We discover location of the essential spectrum for all $\alpha$ and for some cases we establish new properties of the lower bound of this spectrum, which are useful for study of the discrete spectrum.
\end{abstract}

Key words: pseudorelativistic Hamiltonian; many-particle system; permutational symmetry; essential spectrum

2000 Mathematics Subject Classification: 35P20; 35Q75; 46N50; 47N50; 70H05; 81Q10

In this paper we formulate our results on the essential spectrum of many-particle pseudorelativistic Hamiltonians without magnetic and external potential fields in spaces of functions, having arbitrary type $\alpha$ of the permutational symmetry. We discover the location of the essential spectrum for all $\alpha$ (Theorem 1) and for some cases we establish new properties of the lower bound of this spectrum, which are useful for study of the discrete spectrum (Lemma 1).

Before this work similar results on the essential spectrum were obtained in [1,2], but in [2] not arbitrary $\alpha$ were considered, and the construction of the operator of the relative motion was not invariant with respect to the permutations of identical particles in contrast to our approach (in this respect connection of our results with [2] is the same, as connection [5] with [7]); in [1] more extensive class of pseudorelativistic Hamiltonians was studied as compared to [2] and to this paper, but in [1] the permutational symmetry was not considered. Moreover, our Lemma 1 is new.

1. Let $Z_{1}=\{0,1, \ldots, n\}$ be the quantum system of $(n+1)$ particles, $m_{i}, r_{i}=\left(x_{i}, y_{i}, z_{i}\right)$ and $p_{i}$ be the mass, the radius-vector and the momentum of $i$-th particle. Pseudorelativistic (PR) energy operator of $Z_{1}$ can be written in the form

$$
\mathcal{H}^{\prime}=K^{\prime}(r)+V(r),
$$

where $r=\left(r_{0}, r_{1}, \ldots, r_{n}\right)$,

$$
K^{\prime}(r)=\sum_{j=0}^{n}{\sqrt{-\Delta_{j}+m_{j}^{2}}}^{1}, \quad V(r)=V_{0}(r)=\frac{1}{2} \sum_{i, j=0, i \neq j}^{n} V_{i j}\left(\left|r_{i j}\right|\right),
$$

$\Delta_{j}=\frac{\partial^{2}}{\partial x_{j}^{2}}+\frac{\partial^{2}}{\partial y_{j}^{2}}+\frac{\partial^{2}}{\partial z_{j}^{2}}, V_{i j}\left(\left|r_{i j}\right|\right)=V_{j i}\left(\left|r_{j i}\right|\right)$ be the real potential of the interaction $i$-th and $j$-th particles, $r_{i j}=r_{i}-r_{j}, V_{i j}\left(\left|r_{1}\right|\right) \in \mathcal{L}_{2, \mathrm{loc}}\left(\mathbb{R}^{3}\right), V_{i j}\left(\left|r_{1}\right|\right) \rightarrow 0$ at $\left|r_{1}\right| \rightarrow \infty$, and $V_{i j}\left(\left|r_{i j}\right|\right)$ are such

\footnotetext{
${ }^{1}$ We have chosen the unit system so the Plank constant and the light velocity are equal to 1.
} 
that for some $\varepsilon_{0}>0$ operator $\mathcal{H}^{\prime}$ is semibounded from below for $V(r)=\left(1+\varepsilon_{0}\right) V_{0}(r)$. If the system $Z_{1}$ is a molecule, the last condition means that we may consider only the molecules consisting of atoms of such elements whose number in Mendeleev periodic table is smaller than $85[2,3]$.

The operator $\mathcal{H}^{\prime}$ is not local: in the coordinate space operators $\sqrt{-\Delta_{j}+m_{j}^{2}}$ are integral operators, in the momentum representation multiplicators $V_{i j}\left(\left|r_{i j}\right|\right)$ turn into integral operators. But in the momentum space the operators $\sqrt{-\Delta_{j}+m_{j}^{2}}$ are multiplication operators. Actually, let $p_{j}=\left(p_{j 1}, p_{j 2}, p_{j 3}\right), p=\left(p_{0}, \ldots, p_{n}\right), \varphi(r) \in \mathcal{L}_{2}\left(\mathbb{R}^{3 n+3}\right)$, and $\bar{\varphi}(p)$ be Fourier-transform of $\varphi(r)$ :

$$
\bar{\varphi}(p)=\frac{1}{(\sqrt{2 \pi})^{3 n+3}} \int_{\mathbb{R}^{3 n+3}} \varphi(r) e^{i(p, r)} d r,
$$

then

$$
\sqrt{-\Delta_{j}+m_{j}^{2}} \varphi(r)=\sqrt{p_{j}^{2}+m_{j}^{2}} \bar{\varphi}(p) .
$$

Let

$$
T_{j}^{\prime}\left(p_{j}\right)=\sqrt{p_{j}^{2}+m_{j}^{2}}, \quad T^{\prime}(p)=\sum_{j=0}^{n} T_{j}^{\prime}\left(p_{j}\right) .
$$

Now we can rewrite operators $\mathcal{H}^{\prime}$ using mixed form writing:

$$
\mathcal{H}^{\prime}=T^{\prime}(p)+V(r)
$$

where operators $T^{\prime}(p)$ and $V(r)$ act in the momentum and in the coordinate spaces respectively.

2. The operator $\mathcal{H}^{\prime}$ corresponds to the energy of the whole system motion. But for applications it is interesting to know the spectrum of the operator corresponding to the relative motion energy. To get such operator for nonrelativistic (NR) case one separate the center-ofmass motion, but for pseudorelativistic (PR) case it is impossible. To construct the operator of the relative motion from $\mathrm{PR}$ operator $\mathcal{H}^{\prime}$, we reduce the operator $\mathcal{H}^{\prime}$ to any fixed eigenspace of operator of the total momentum [2]. Let $\xi_{0}=\left(\xi_{01}, \xi_{02}, \xi_{03}\right)$ be the center-of-mass radius-vector:

$$
\xi_{0}=\sum_{j=0}^{n} m_{j} r_{j} / M, \quad M=\sum_{j=0}^{n} m_{j}
$$

$q_{j}=r_{j}-\xi_{0}$ be the relative coordinates of $j$-th particle, $j=0,1, \ldots, n, q=\left(q_{0}, \ldots, q_{n}\right)$. We take $q, \xi_{0}$ as the new coordinates of the particles from $Z_{1}$. Let us note that vectors $q_{0}, \ldots, q_{n}$ are dependent: they belong to the space

$$
R_{0}=\left\{q^{\prime} \mid q^{\prime}=\left(q_{0}^{\prime}, \ldots, q_{n}^{\prime}\right), \quad \sum_{j=0}^{n} m_{j} q_{j}^{\prime}=\theta=(0,0,0)\right\}
$$

of relative motion. On the other hand, if $q^{\prime}=\left(q_{0}^{\prime}, \ldots, q_{n}^{\prime}\right) \in R_{0}$ and $\xi_{0}^{\prime}$ is an arbitrary fixed vector from $\mathbb{R}^{3}$, we may consider $q_{j}^{\prime}$ and $\xi_{0}^{\prime}$ as the relative coordinates of the point $r_{j}^{\prime}=q_{j}^{\prime}+\xi_{0}^{\prime}$, $j=0,1, \ldots, n$ and the center-of-mass position of $Z_{1}$ respectively. It is easy to see that Fourierconjugate coordinates to $q_{j}$ are the same $p_{j}$ as for $r_{j}$, and Fourier-conjugate coordinate for $\xi_{0}$ is $\mathcal{P}_{0}=\left(\mathcal{P}_{01}, \mathcal{P}_{02}, \mathcal{P}_{03}\right)=\sum_{j=0}^{n} p_{j}$

Let us consider the operators

$$
L_{0 s}=\frac{1}{i} \frac{d}{d \xi_{0 s}}, \quad s=1,2,3 .
$$


In the momentum space these operators are multiplication operators

$$
\bar{L}_{0 s}=\mathcal{P}_{0 s} .
$$

It follows from above that the operators $L_{0 s}\left\{\bar{L}_{0 s}\right\}$ commute with $\mathcal{H}^{\prime}$. So any eigenspaces of the operators $L_{0 s}$ are invariant for $\mathcal{H}^{\prime}$. Let $-Q_{0 s}$ be a real eigenvalue of the operator $L_{0 s}, W_{0 s}$ be corresponding eigenspace and

$$
W_{0}=W_{01} \cap W_{02} \cap W_{03} .
$$

The space $W_{0}$ is invariant for $\mathcal{H}^{\prime}$. Evidently

$$
W_{0}=\left\{(2 \pi)^{-3 / 2} e^{-i\left(Q_{0}, \xi_{0}\right)} \varphi(q)\right\}^{2}, \quad \bar{W}_{0}=\left\{\bar{\varphi}(p) \prod_{s=1}^{3} \delta\left(\mathcal{P}_{0 s}-Q_{0 s}\right)\right\},
$$

where $Q_{0}=\left(Q_{01}, Q_{02}, Q_{03}\right), \varphi(q)$ is an arbitrary function, $\varphi(q) \in \mathcal{L}_{2}\left(R_{0}\right)$, and $\bar{W}_{0}$ is Fourierimage of $W_{0}$.

Let us rewrite operator $\mathcal{H}^{\prime}$ using the coordinates $q, \xi_{0}\{p, \mathcal{P}\}$ and reduce it to the subspace $W_{0}\left\{\bar{W}_{0}\right\}$. Then we obtain the operator $\mathcal{H}^{\prime}$ in the form

$$
H_{0}^{\prime}=T^{\prime}\left(p, Q_{0}\right)+V(q),
$$

where

$$
T^{\prime}\left(p, Q_{0}\right)=T^{\prime}(p),
$$

but with the condition

$$
\begin{aligned}
& \sum_{j=0}^{n} p_{j}=Q_{0} \\
& V(q)=\frac{1}{2} \sum_{i, j=0, i \neq j}^{n} V_{i j}\left(\left|q_{i}-q_{j}\right|\right), \quad q_{i}-q_{j}=r_{i}-r_{j} .
\end{aligned}
$$

We see that $H_{0}^{\prime}$ depends on the relative coordinates $q$, their momenta $p$ and the total momentum value $Q_{0}$. So if we fix $Q_{0}$ we obtain the operator, which can be considered as the operator of the relative motion. We shall study this operator in the space $\mathcal{L}_{2}\left(R_{0}\right)$ with condition (1) for momenta.

For technical reasons it is convenient to take

$$
T_{j}\left(p_{j}\right)=T_{j}^{\prime}\left(p_{j}\right)-m_{j}
$$

instead of $T_{j}^{\prime}\left(p_{j}\right)$ and

$$
T\left(p, Q_{0}\right)=\sum_{j=0}^{n} T_{j}\left(p_{j}\right)
$$

instead of $T^{\prime}\left(p ; Q_{0}\right)$. So the subject of our study is operator

$$
H_{0}=T\left(p ; Q_{0}\right)+V(q)
$$

\footnotetext{
${ }^{2}$ The coefficient $(2 \pi)^{-3 / 2}$ in front of the $e^{-i\left(Q_{0}, \xi_{0}\right)}$ plays the role of "normalizing factor": Fourier-image of $(2 \pi)^{-3 / 2} e^{-i\left(Q_{0}, \xi_{0}\right)}$ is $\prod_{s=1}^{s} \delta\left(\mathcal{P}_{0 s}-Q_{0 s}\right)$ without any factor.
} 
(with condition (1)). The operator $H_{0}$ is bounded from below on $C_{0}^{\infty}\left(R_{0}\right)$. We extend it to a self-adjoint one using Friedrichs extension, and save the notation $H_{0}$ for the obtained operator.

Let us note that instead of the dependent coordinates $q_{0}, \ldots, q_{n}$ we could introduce independent relative coordinates (and their momenta) similar to [2], but such approach generates difficulties, when one takes into account the permutational symmetry (see $\S \mathbf{5}$ ), and we do not use this approach.

3. We shall study spectrum of the operator $H_{0}$ not in the whole space $\mathcal{L}_{2}\left(R_{0}\right)$, but in the subspaces of functions from $\mathcal{L}_{2}\left(R_{0}\right)$, having the fixed types of permutational symmetry. We do this

i) to satisfy the Pauli exclusion principle,

ii) to obtain additional information about the structure of the spectrum $H_{0}$.

We denote by $S$ and $\alpha$ the group of the permutations of all identical particles of $Z_{1}$ and an arbitrary type of irreducible representation of $S$ respectively. Let us determine the operators $T_{g}$, $g \in S$ by relations

$$
T_{g} \varphi(q)=\varphi\left(g^{-1} q\right), \quad g \in S
$$

and put

$$
P^{(\alpha)}=\frac{l_{\alpha}}{|S|} \sum_{g \in S} \bar{\chi}_{g}^{(\alpha)} T_{g}, \quad B^{(\alpha)}=P^{(\alpha)} \mathcal{L}_{2}\left(R_{0}\right),
$$

where $\chi_{g}^{(\alpha)}$ is the character of the element $g \in S$ in the irreducible representation of the type $\alpha$, $l_{\alpha}$ is the dimension of this representation, $|S|$ is the number of elements of $S$. The operator $P^{(\alpha)}$ is the projector in $\mathcal{L}_{2}\left(R_{0}\right)$ on the subspace $B^{(\alpha)}=B^{(\alpha)}\left(R_{0}\right)$ of functions, which are transformed by the operators $T_{g}, g \in S$, according to the representation of the type $\alpha$ [6]. Evidently $P^{(\alpha)} H_{0}=$ $H_{0} P^{(\alpha)}$. Let $H_{0}^{(\alpha)}=H_{0} P^{(\alpha)}$. $H_{0}^{(\alpha)}$ be the restriction of the operator $H_{0}$ to the subspace $B^{(\alpha)}$ of functions, having the permutational symmetry of the type $\alpha$.

In this paper we discover location of the essential spectrum $s_{\text {ess }}\left(H_{0}^{(\alpha)}\right)$ of the operator $H_{0}^{(\alpha)}$.

4. Let $Z_{2}=\left(D_{1}, D_{2}\right)$ be an arbitrary decomposition of the initial system $Z_{1}$ into 2 non-empty clusters $D_{1}$ and $D_{2}$ without common elements:

$$
D_{1} \cup D_{2}=Z_{1}, \quad D_{1} \cap D_{2}=\varnothing
$$

and

$$
H\left(Z_{2}\right)=T\left(p, Q_{0}\right)+V\left(q ; Z_{2}\right)
$$

where

$$
V\left(q ; Z_{2}\right)=\frac{1}{2} \sum_{s=1}^{2} \sum_{i, j \in D_{s}, i \neq j} V_{i j}\left(\left|q_{j}-q_{i}\right|\right) .
$$

$H\left(Z_{2}\right)$ is the PR energy operator of compound system $Z_{2}$, consisting of non interacting (one with other) clusters $D_{1}, D_{2}$ with the same condition (1) for the total momentum as for $Z_{1}$ :

$$
\sum_{i=0}^{n} p_{i}=Q_{0}
$$


Let $S\left[D_{s}\right]$ be the group of the permutations of all identical particles from $D_{s}, s=1,2, \hat{g}$ be the permutation $D_{1} \leftrightarrow D_{2}$ if these clusters are identical $\left(D_{1} \sim D_{2}\right)$. We put

$$
\begin{aligned}
& S_{0}\left(Z_{2}\right)=S\left[D_{1}\right] \times S\left[D_{2}\right], \\
& S\left(Z_{2}\right)=S_{0}\left(Z_{2}\right) \quad \text { if } \quad D_{1} \nsim D_{2}, \\
& S\left(Z_{2}\right)=\hat{S}\left(Z_{2}\right)=S_{0}\left(Z_{2}\right) \cup S_{0}\left(Z_{2}\right) \hat{g} \quad \text { if } \quad D_{1} \sim D_{2} .
\end{aligned}
$$

$S\left(Z_{2}\right)$ is the group of the permutational symmetry of the compound system $Z_{2}$. It is clear that $S_{0}\left(Z_{2}\right) \subseteq S\left(Z_{2}\right) \subseteq S$.

Let $F\left(\alpha ; Z_{2}\right)=\left\{\alpha^{\prime}\right\}\left\{F_{0}\left(\alpha ; Z_{2}\right)=\{\check{\alpha}\}\right\}$ be the set of all types $\alpha^{\prime}\{\check{\alpha}\}$ of the group $S\left(Z_{2}\right)\left\{S_{0}\left(Z_{2}\right)\right\}$ irreducible representations, which are contained in the group $S$ irreducible representation $D_{g}^{(\alpha)}$ of the type $\alpha$ after reducing $D_{g}^{(\alpha)}$ from $S$ to $S\left(Z_{2}\right)\left\{S_{0}\left(Z_{2}\right)\right\}$. For $\forall \alpha^{\prime}\{\check{\alpha}\}$ we determine the projector $P^{\left(\alpha^{\prime}\right)}\left(Z_{2}\right)\left\{P^{(\check{\alpha})}\left(Z_{2}\right)\right\}$ on the subspace of functions $\varphi(q)$, which are transformed by operators $T_{g}$

$$
T_{g} \varphi(q)=\varphi\left(g^{-1} q\right), \quad g \in S\left(Z_{2}\right), \quad\left\{g \in S_{0}\left(Z_{2}\right)\right\}
$$

according to the group $S\left(Z_{2}\right)\left\{S_{0}\left(Z_{2}\right)\right\}$ irreducible representation of the type $\alpha^{\prime}\{\check{\alpha}\}$.

Let $\gamma=\alpha^{\prime}$ or $\gamma=\check{\alpha}$; obviously if $P^{(\gamma)}\left(Z_{2}\right) \varphi(q)=\varphi(q)$, then $P^{(\gamma)}\left(Z_{2}\right) \bar{\varphi}(p)=\bar{\varphi}(p)$. We set

$$
\begin{aligned}
& P\left(\alpha ; Z_{2}\right)=\sum_{\alpha^{\prime} \in F\left(\alpha ; Z_{2}\right)} P^{\left(\alpha^{\prime}\right)}\left(Z_{2}\right), \quad \check{P}\left(\alpha ; Z_{2}\right)=\sum_{\check{\alpha} \in F_{0}\left(\alpha ; Z_{2}\right)} P^{(\check{\alpha})}\left(Z_{2}\right), \\
& H\left(\alpha ; Z_{2}\right)=H\left(Z_{2}\right) P\left(\alpha ; Z_{2}\right), \quad \check{H}\left(\alpha ; Z_{2}\right)=H\left(Z_{2}\right) \check{P}\left(\alpha ; Z_{2}\right) .
\end{aligned}
$$

The operator $H\left(\alpha ; Z_{2}\right)\left\{\check{H}\left(\alpha ; Z_{2}\right)\right\}$ is the restriction of the operator $H\left(Z_{2}\right)$ (see (3)) to the subspace $B\left(\alpha ; Z_{2}\right)=P\left(\alpha ; Z_{2}\right) \mathcal{L}_{2}\left(R_{0}\right)\left\{\check{B}\left(\alpha ; Z_{2}\right)=\check{P}\left(\alpha ; Z_{2}\right) \mathcal{L}_{2}\left(R_{0}\right)\right\}$. Let

$$
\mu^{(\alpha)}=\min _{Z_{2}} \inf H\left(\alpha ; Z_{2}\right) .
$$

It is possible to prove that

$$
\mu^{(\alpha)}=\min _{Z_{2}} \inf \check{H}\left(\alpha ; Z_{2}\right) .
$$

We denote by $A(\alpha)$ the set of all $Z_{2}$, for which

$$
\inf \check{H}\left(\alpha ; Z_{2}\right)=\min _{Z_{2}^{\prime}} \inf \check{H}\left(\alpha ; Z_{2}^{\prime}\right) ;
$$

then

$$
\mu^{(\alpha)}=\inf \check{H}\left(\alpha ; Z_{2}\right), \quad Z_{2} \in A(\alpha) .
$$

5. Our main result is the following theorem

Theorem 1. Essential spectrum $s_{\mathrm{ess}}\left(H_{0}^{(\alpha)}\right)$ of the operator $H_{0}^{(\alpha)}$ consists of all points half-line $\left[\mu^{(\alpha)},+\infty\right)$.

Let us compare Theorem 1 with the corresponding results in [2].

First, in [2] a similar result was proved only for one of simplest types $\alpha$ of the permutational symmetry (for $\alpha$ corresponding to one-column Young scheme), while here we assume arbitrary $\alpha$.

Second, we use more natural, simple and transparent approach for taking symmetry into account, compared to [2]. Actually, we apply relative coordinates $q_{i}$ with respect to center-ofmass position $\xi_{0}: q_{i}=r_{i}-\xi_{0}, i=0,1, \ldots, n$ and so the transposition $g_{j}: r_{j} \leftrightarrow r_{0}$ of $j$-th and 
0 -th particles results in the transposition of $q_{j}$ and $q_{0}$ only, but just as all other coordinates $q_{i}$, $i \neq j, i \neq 0$, are without any change. In [2] relative coordinates $\tilde{q}_{i}$ are taken with respect to the position of 0 -th particle: $\tilde{q}_{i}=r_{i}-r_{0}, i=1,2, \ldots, n$ and this choice implies changing of all $\tilde{q}_{i}$ under transposition $g_{j}$. Namely, $T_{g_{j}} \psi(\tilde{q})=\psi\left(g_{j}^{-1} \tilde{q}\right)=\psi(\hat{q})$, where $\tilde{q}=\left(\tilde{q}_{1}, \ldots, \tilde{q}_{n}\right)$, $\hat{q}=\left(\hat{q}_{1}, \ldots \hat{q}_{n}\right), \hat{q}_{i}=\tilde{q}_{i}-\tilde{q}_{j}, i \neq j, \hat{q}_{j}=-\tilde{q}_{j}$. Such situation is not realized only if the system $Z_{1}$ contains a particle, which is not identical to any other particle from $Z_{1}$ (and if we index this particle by number 0 ), but there is no such exceptional particle in the most number of molecules. Completing the second remark, we can note, roughly speaking, that our approach for taking permutational symmetry into account follows [5], while authors [2] follow [7].

6. We do not write here the proof of the Theorem 1 , since the significant part of this proof will be needed for the study of the discrete spectrum $s_{d}\left(H_{0}^{(\alpha)}\right)$ of the operator $H_{0}^{(\alpha)}$ (this study is not finished), so we shall publish the full proof of the Theorem 1 later (together with the results on the discrete spectrum). But here we shall do some preparations for our next paper. Namely, we shall obtain from (4), (5) the other formula for $\mu^{(\alpha)}$, which is more convenient for the investigation of the structure $s_{d}\left(H_{0}^{(\alpha)}\right)$. To do it first of all we transform the expression of the operator $H\left(Z_{2}\right)$ for fixed $Z_{2}=\left(D_{1}, D_{2}\right)$. We introduce clusters $D_{s}$ center-of-mass coordinates

$$
\xi_{s}=\left(\xi_{s 1}, \xi_{s 2}, \xi_{s 3}\right)=\sum_{j \in D_{s}} r_{j} m_{j} / M_{s}, \quad M_{s}=\sum_{j \in D_{s}} m_{j}
$$

the relative coordinates $q_{j}\left(Z_{2}\right)=r_{j}-\xi_{s}, j \in D_{s}$, of the particles from $D_{s}$ with respect to center-of-mass position and the vector $\eta=\xi_{2}-\xi_{1}$. Evidently, $q_{j}\left(Z_{2}\right)=q_{j}+\xi_{0}-\xi_{s}$, where $\xi_{0}-\xi_{1}=M_{2} \eta / M, \xi_{0}-\xi_{2}=-M_{1} \eta / M$.

The coordinates $q\left(Z_{2}\right)=\left(q_{0}\left(Z_{2}\right), \ldots, q_{n}\left(Z_{2}\right)\right)$ are not independent, since $\sum_{j \in D_{s}} m_{j} q_{j}\left(Z_{2}\right)=\theta$, $s=1,2$. It is easy to see that Fourier-conjugate coordinates to $q_{j}\left(Z_{2}\right)$ are the same $p_{j}$ that were introduced before. Let $\mathcal{P}_{s}=\sum_{j \in D_{s}} p_{j}$. Then Fourier-conjugate coordinates to $\eta$ are

$$
\mathcal{P}_{\eta}=\left(\mathcal{P}_{\eta 1}, \mathcal{P}_{\eta 2}, \mathcal{P}_{\eta 3}\right)=\left(\mathcal{P}_{2} M_{1}-\mathcal{P}_{1} M_{2}\right) / M
$$

where by (1)

$$
\mathcal{P}_{1}+\mathcal{P}_{2}=Q_{0}
$$

We consider $q\left(Z_{2}\right)$ and $\eta$ as new coordinates of particles from $Z_{1}$ and denote the operator $H\left(Z_{2}\right)$ in new coordinates by $H_{0}\left(Z_{2}\right)$. According to consideration above and since $q_{i}-q_{j}=$ $q_{i}\left(Z_{2}\right)-q_{j}\left(Z_{2}\right), i, j \in D_{s}, s=1,2$, we have

$$
H\left(Z_{2}\right)=H_{0}\left(Z_{2}\right)=T\left(p, Q_{0}, \mathcal{P}_{\eta}\right)+V\left(q\left(Z_{2}\right) ; Z_{2}\right)
$$

where the operator (7) has the same form as the operator (3), but the conditions (6) have to be satisfied.

Let us introduce spaces

$$
\begin{aligned}
& R_{0}\left(Z_{2}\right)=\left\{q\left(Z_{2}\right) \mid q\left(Z_{2}\right)=\left(q_{0}\left(Z_{2}\right), \ldots, q_{n}\left(Z_{2}\right)\right), \quad \sum_{j \in D_{s}} m_{j} q_{j}\left(Z_{2}\right)=\theta, \quad s=1,2\right\}, \\
& R_{\eta}=\left\{\eta \mid \eta=\left(\eta_{1}, \eta_{2}, \eta_{3}\right)\right\}, \quad R_{0, \eta}\left(Z_{2}\right)=R_{0}\left(Z_{2}\right) \oplus R_{\eta}, \\
& \mathcal{L}_{2}\left(R_{0, \eta}\left(Z_{2}\right)\right)=\left\{\left.\varphi\left(q\left(Z_{2}\right), \eta\right)\left|\int_{R_{0, \eta}}\right| \varphi\right|^{2} d q\left(Z_{2}\right) d \eta<+\infty\right\} .
\end{aligned}
$$


In the space $\mathcal{L}_{2}\left(R_{0, \eta}\left(Z_{2}\right)\right)$ we determine operators $P_{0}^{(\check{\alpha})}\left(Z_{2}\right)$ similarly to operators $P^{(\check{\alpha})}\left(Z_{2}\right)$, but now the operators $T_{g}, g \in S_{0}\left(Z_{2}\right)$, are defined on functions $\varphi\left(q\left(Z_{2}\right), \eta\right)$ and $\bar{\varphi}\left(p, \mathcal{P}_{\eta}\right)$ by relations

$$
T_{g} \varphi\left(q\left(Z_{2}\right), \eta\right):=\varphi\left(g^{-1} q\left(Z_{2}\right), \eta\right), \quad T_{g} \bar{\varphi}\left(p, \mathcal{P}_{\eta}\right)=\bar{\varphi}\left(g^{-1} p, \mathcal{P}_{\eta}\right) .
$$

Here we took into account that $g^{-1} \eta=\eta$ and $g^{-1} \mathcal{P}_{\eta}=\mathcal{P}_{\eta}$ for $\forall \eta, \mathcal{P}_{\eta}, \forall g \in S_{0}\left(Z_{2}\right)$.

Let us

$$
\check{P}_{0}\left(\alpha ; Z_{2}\right)=\sum_{\check{\alpha} \in F_{0}\left(\alpha ; Z_{2}\right)} P_{0}^{(\check{\alpha})}\left(Z_{2}\right), \quad \check{H}_{0}\left(\alpha ; Z_{2}\right)=H_{0}\left(Z_{2}\right) \check{P}_{0}\left(\alpha ; Z_{2}\right) .
$$

According to (5),

$$
\mu^{(\alpha)}=\inf \check{H}_{0}\left(\alpha ; Z_{2}\right), \quad Z_{2} \in A(\alpha),
$$

where the operator $\check{H}_{0}\left(\alpha ; Z_{2}\right)$ is considered in the space $\mathcal{L}_{2}\left(R_{0, \eta}\right)$. Since the operator $T\left(p, Q_{0}, \mathcal{P}_{\eta}\right)$ is a multiplication operator and the potential $V\left(q\left(Z_{2}\right) ; Z_{2}\right)$ does not depend on $\eta$, we may consider the operator $\check{H}_{0}\left(\alpha ; Z_{2}\right) \equiv \check{H}_{0}\left(\alpha ; Z_{2} ; \mathcal{P}_{\eta}\right)$ in the space $\mathcal{L}_{2}\left(R_{0}\left(Z_{2}\right)\right)$ at the arbitrary fixed $\mathcal{P}_{\eta}=Q$. Then

$$
\mu^{(\alpha)}=\inf _{Q} \inf \check{H}_{0}\left(\alpha ; Z_{2} ; Q\right), \quad Z_{2} \in A(\alpha) .
$$

Operator $\check{H}\left(\alpha ; Z_{2} ; Q\right)$ depends on $Q$ continuously and

$$
\lim _{|Q| \rightarrow+\infty} \inf \check{H}_{0}\left(\alpha ; Z_{2} ; Q\right)=+\infty
$$

since if $|Q| \rightarrow+\infty$, then at least for one $j$ it holds $\left|p_{j}\right| \rightarrow \infty$ and consequently $T\left(p, Q_{0}, Q\right) \rightarrow+\infty$. So there exists a compact set $\Gamma\left(\alpha ; Z_{2}\right)$ of such vectors $Q \in \mathbb{R}^{3}$ that

$$
\mu^{(\alpha)}=\inf \check{H}_{0}\left(\alpha ; Z_{2} ; Q\right), \quad Q \in \Gamma\left(\alpha ; Z_{2}\right), \quad Z_{2} \in A(\alpha) .
$$

7. Unfortunately, in the general case we know nothing about finiteness or infiniteness of the number of the set $\Gamma\left(\alpha ; Z_{2}\right)$ elements. But we can prove the following assertion

Lemma 1. Let for some open region $W \subset \mathbb{R}^{3}, \Gamma\left(\alpha ; Z_{2}\right) \subset W$,

i) $\lambda\left(\alpha ; Z_{2} ; Q\right):=\inf \check{H}_{0}\left(\alpha ; Z_{2} ; Q\right)$ is the point of the discrete spectrum of the operator $\check{H}_{0}\left(\alpha ; Z_{2} ; Q\right)$ for $Q \in W$,

ii) there is such $\check{\alpha}_{0}$, which does not depend on $Q$, that the representation $g \rightarrow T_{g}, g \in S_{0}\left(Z_{2}\right)$ in the eigenspace $U\left(\alpha ; Z_{2} ; Q\right)$ of the operator $\check{H}_{0}\left(\alpha ; Z_{2} ; Q\right)$, corresponding to its eigenvalue $\lambda\left(\alpha ; Z_{2} ; Q\right)$, has ONE irreducible component of the type $\check{\alpha}_{0}$ for each $Q \in W$.

Then the set $\Gamma\left(\alpha ; Z_{2}\right)$ is finite.

Proof. Let $\check{B}_{0}\left(\alpha ; Z_{2}\right)=\check{P}_{0}\left(\alpha ; Z_{2}\right) \mathcal{L}_{2}\left(R_{0}\left(Z_{2}\right)\right)$. Since

$$
\check{P}_{0}\left(\alpha ; Z_{2}\right)=P_{0}^{\left(\check{\alpha}_{0}\right)}\left(Z_{2}\right)+\sum_{\check{\alpha} \in F_{0}\left(\alpha ; Z_{2}\right), \check{\alpha} \neq \check{\alpha}_{0}} P_{0}^{(\check{\alpha})}\left(Z_{2}\right),
$$

then

$$
B_{0}^{\left(\check{\alpha}_{0}\right)}\left(Z_{2}\right):=P_{0}^{\left(\check{\alpha}_{0}\right)}\left(Z_{2}\right) \check{B}_{0}\left(\alpha ; Z_{2}\right)=P_{0}^{\left(\check{\alpha}_{0}\right)}\left(Z_{2}\right) \mathcal{L}_{2}\left(R_{0}\left(Z_{2}\right)\right) .
$$


It follows from the conditions i), ii) that in the space

$$
U^{\left(\check{\alpha}_{0}\right)}=U\left(\alpha ; Z_{2} ; Q\right) \cap B_{0}^{\left(\check{\alpha}_{0}\right)}\left(Z_{2}\right) \equiv P_{0}^{\left(\check{\alpha}_{0}\right)} U\left(\alpha ; Z_{2} ; Q\right)
$$

the representation $g \rightarrow T_{g}, g \in S_{0}\left(Z_{2}\right)$ is irreducible and has the type $\check{\alpha}_{0}$.

Let $P_{01}^{\left(\check{\alpha}_{0}\right)}$ be the projector in $B_{0}^{\left(\check{\alpha}_{0}\right)}\left(Z_{2}\right)$ on the space $B_{01}^{\left(\check{\alpha}_{0}\right)}\left(Z_{2}\right)$ of functions, which belong to the first line of the group $S_{0}\left(Z_{2}\right)$ irreducible representation of the type $\check{\alpha}_{0}$.

Then the space $B_{01}^{\left(\check{\alpha}_{0}\right)}\left(Z_{2}\right)$ is invariant under the operator $H_{0}\left(Z_{2}\right)$ and in this space the minimal eigenvalue $\lambda\left(\alpha ; Z_{2} ; Q\right)$ of the operator $H_{0}\left(Z_{2}\right)$ is nondegenerated, since the corresponding eigenspace $P_{01}^{\left(\check{\alpha}_{0}\right)} U^{\left(\check{\alpha}_{0}\right)}$ is one-dimensional. In other words, the minimal eigenvalue of the operator $P_{01}^{\left(\check{\alpha}_{0}\right)} H_{0}\left(\alpha ; Z_{2} ; Q\right)$ is nondegenerated at $\forall Q \in W$. But if $\lambda\left(\alpha ; Z_{2} ; Q\right)$ is nondegenerated, then $\lambda\left(\alpha ; Z_{2} ; Q\right)$ is analytical function of $Q$, since the operator $H_{0}\left(Z_{2}\right)$ is analytical function on $Q$ [4]. That is why there is only finite number of such vectors $Q$, for which

$$
\mu^{(\alpha)}=\lambda\left(\alpha ; Z_{2} ; Q\right) .
$$

8. Discussion. Theorem 1 and Lemma 1 describe the location of essential spectrum $s_{\text {ess }}\left(H_{0}^{(\alpha)}\right)$ of the operator $H_{0}^{(\alpha)}$ and some properties of its lower bound respectively. Now let us consider a role of these results for the discrete spectrum study. It follows from Theorem 1 that to prove the existence of nonempty discrete spectrum $s_{d}\left(H_{0}^{(\alpha)}\right)$ of the operator $H_{0}^{(\alpha)}$ it is sufficient to construct such trial function $\psi, P^{(\alpha)} \psi=\psi$ that

$$
\left(H_{0}^{(\alpha)} \psi, \psi\right)<\mu^{(\alpha)}(\psi, \psi)
$$

where the number $\mu^{(\alpha)}$ is determined by the relations (5) and (8). Construction of a function $\psi$ for (9) is important component of geometrical methods application in the study of the spectrum $s_{d}\left(H_{0}^{(\alpha)}\right)$ of operator $H_{0}^{(\alpha)}$.

But Theorem 1 is not a sufficient base to study the spectral asymptotics of the discrete spectrum $s_{d}\left(H_{0}^{(\alpha)}\right)$ near $\mu^{(\alpha)}$, when this spectrum is infinite. To understand the reason for that, let us consider the case when $\mu^{(\alpha)}$ is the point of the spectrum $s_{d}\left(H\left(\alpha ; Z_{2} ; Q\right)\right)$ for $Z_{2} \in$ $A(\alpha), Q \in \Gamma\left(\alpha ; Z_{2}\right)$ (such situation is expected for $\mathrm{PR}$ atoms). Then the infinite series of the eigenvalues $\lambda_{k}(Q), k=1,2, \ldots$, from $s_{d}\left(H_{0}^{(\alpha)}\right)$ may exist for $\forall Q \in \Gamma\left(\alpha ; Z_{2}\right)$. In this case it is possible to show that corresponding eigenfunctions $\psi_{k}$ describe (when $k \rightarrow \infty$ ) such decomposition $Z_{2}=\left\{C_{1}, C_{2}\right\}$ of the initial system $Z_{1}$, for which

$$
\mathcal{P}_{1}+\mathcal{P}_{2}=Q_{0}, \quad M_{1} \mathcal{P}_{2}-M_{2} \mathcal{P}_{1}=M Q
$$

(see (6)). Consequently, if the set $\Gamma\left(\alpha ; Z_{2}\right)$ is infinite, then the spectrum $s_{d}\left(H_{0}^{(\alpha)}\right)$ may consist of infinite number of the infinite series eigenvalues $\lambda_{k}(Q), k=1,2, \ldots$, where all series are determined by the values $Q$ from $\Gamma\left(\alpha ; Z_{2}\right)$. For such situation there are no approaches to get the spectral asymptotics of $s_{d}\left(H_{0}^{(\alpha)}\right)$. Thus, it was very desirable to establish the conditions of impossibility of this situation that is the conditions of finiteness of the set $\Gamma\left(\alpha ; Z_{2}\right)$. Namely, such conditions are given in Lemma 1 of the paper.

\section{Acknowledgements}

This investigation is supported by RFBR grant 05-01-00299. 
[1] Damak M., On the spectral theory of dispersive N-body Hamiltonians, J. Math. Phys., 1999, V.40, 35-48.

[2] Lewis R.T., Siedentop H., Vugalter S., The essential spectrum of relativistic multi-particle operators, Ann. Inst. H. Poincaré Phys. Théor., 1997, V.67, 1-28.

[3] Lieb E., Yau H.-T., The stability and instability of relativistic matter, Comm. Math. Phys., 1988, V.118, $177-213$.

[4] Reed M., Simon B., Methods of modern mathematical physics. IV Analysis of operators, New York - San Francisco - London, Academic Press, 1978.

[5] Sigalov A.G., Sigal I.M., Invariant description, with respect to transpositions of identical particles, of the energy operator spectrum of quantum-mechanical systems, Teoret. Mat. Fiz., 1970, V.5, 73-93 (in Russian).

[6] Wigner E.P., Group theory and its application to quantum mechanics, New York, 1959.

[7] Zhislin G., Spectrum of differential operators of quantum mechanical many-particle system in the spaces of functions of the given symmetry, Izvest. Akad. Nauk SSSR, Ser. Mat., 1969, V.33, 590-649 (English transl.: Math. USSR-Izvestia, 1969, V.3, 559-616). 